\section{Proteomic analysis of the pulvinus, a heliotropic tissue, in Glycine max}

\author{
Hakme Lee, ${ }^{1}$ Wesley M. Garrett, ${ }^{2}$ \\ Joseph Sullivan, ${ }^{1}$ Irwin Forseth, ${ }^{1}$ \\ Savithiry S. Natarajan ${ }^{3}$ \\ ${ }^{1}$ Department of Plant Sciences and \\ Landscape Architecture, University of \\ Maryland, College Park, MD; ${ }^{2}$ USDA-ARS, \\ Animal Bioscience and Biotechnology \\ Laboratory, Beltsville, MD; ${ }^{3}$ USDA-ARS, \\ Soybean Genomics and Improvement \\ Laboratory, Beltsville, MD, USA
}

\section{Abstract}

Certain plant species respond to light, dark, and other environmental factors by leaf movement. Leguminous plants both track and avoid the sun through turgor changes of the pulvinus tissue at the base of leaves. Mechanisms leading to pulvinar turgor flux, particularly knowledge of the proteins involved, are not well-known. In this study we used two-dimensional gel electrophoresis and liquid chromatography-tandom mass spectrometry to separate and identify the proteins located in the soybean pulvinus. A total of 183 spots were separated and 195 proteins from 165 spots were identified and functionally analyzed using single enrichment analysis for gene ontology terms. The most significant terms were related to proton transport. Comparison with guard cell proteomes revealed similar significant processes but a greater number of pulvinus proteins are required for comparable analysis. To our knowledge, this is a novel report on the analysis of proteins found in soybean pulvinus. These findings provide a better understanding of the proteins required for turgor change in the pulvinus.

\section{Introduction}

Plants respond to light, gravity, touch, and other environmental signals by both temporary and permanent differential growth. ${ }^{1,2}$ The directional growth of plants as a response to an external stimulus is called tropism. The movement of leaves by sunlight, or heliotropism, can angle leaf lamina both toward (diaheliotropism) and away from (paraheliotropism) the light depending on the intensity of the irradiance, circadian rhythms (nyctinasty), and environmental stresses. Diaheliotropic movement has been shown to increase water use efficiency and carbon assimilation compared to horizontal controls in cotton and soybean., Paraheliotropism benefits plants by maintaining high levels of photosynthetic quantum yield under stressed conditions and reducing UVB radiation levels. ${ }^{5,6}$

The pulvinus is an enlarged motor organ at the base of leaves found in many leguminous plants. It has been observed to force the movement of leaves in heliotropic, seismonastic, and nyctinastic patterns. ${ }^{7,8}$ Unlike pulvini found in maize and oat that respond to gravity by permanent growth, the movement of soy bean pulvini is reversible. ${ }^{8,9}$ In heliotropism, upon light exposure, an asymmetric turgor gradient formed between the adaxial and abaxial motor cells leads to leaf movement. Potassium ion influx coupled with chlorine ion is powered by a proton gradient and results in osmotic influx. ${ }^{7}$ In addition to heliotropism, the pulvinus changes turgor for nyctinastic leaf folding, and is affected by alterations in the length of the photoperiod. ${ }^{10}$

The structure of the pulvinus reveals its specialized role in leaf movement. In contrast to the stem and petiole, the pulvinus has a relatively larger cortex and smaller pith. The motor cells are part of the cortex. ${ }^{11}$ Two types of pulvinar vacuoles found in many species participate in the volume flux. Tannin-rich vacuoles have been previously mentioned as a major source of cellular volume change. ${ }^{12}$ Still other studies have found the primary volume changes to be from the type of vacuole that does not contain tannin. ${ }^{13}$

A number of proteins have been linked with pulvinar heliotropism and nyctinasty. $\mathrm{H}^{+}$ATPase activity increases turgor by $\mathrm{H}^{+}$efflux and consequent $\mathrm{K}^{+}$influx, and $\mathrm{H}^{+}$-ATPase inhibitors reduce diaheliotropic response in soybean pulvinus. ${ }^{14}$ Blue light is a deactivator of $\mathrm{H}^{+}$-ATPase in Phaseolus vulgaris motor cells which leads to decreased turgor pressure on the illuminated region rather than an activator of $\mathrm{H}^{+}$-ATPase on the opposite region. ${ }^{15}$ Furthermore, studies on gravitropic grass pulvini proteins have begun to identify differentially expressed proteins including one demonstrating MAPK-like activity. ${ }^{16}$

While studies have identified a number of genes involved in a variety of tropic responses, there remains a great dearth of knowledge on the gene products and their expression patterns. The purpose of this study was to map the proteome of the soybean (Glycine max) pulvinus using trichloroacetic acid/acetone extraction, 2-dimensional gel electrophoresis (2-DE) and identification by liquid chromatographytandom mass spectrometry (LC-MS/MS). This map would highlight the molecular and functional characterization of the pulvinus at the protein level. The soybean has been selected because of its importance as a food crop, the
Correspondence: Savithiry Natarajan, Soybean Genomics and Improvement Laboratory, ARSUSDA, 10300 Baltimore Avenue, Beltsville, MD 20705, USA.

Tel. +1.301.504.5258 - Fax: +1.301.504.5728.

E-mail: savi.natarajan@ars.usda.gov

Key words: soybean, heliotropism, nyctinasty, proteomics, LC-MS/MS

Acknowledgments: we thank Dr. William Kenworthy for providing the seeds for this project. This work was carried out with the support of a Cooperative Agreement between the University of Maryland, College Park, and USDA-ARS in Beltsville, MD, USA.

Contributions: the authors contributed equally.

Received for publication: 27 May 2013.

Revision received: 19 August 2013.

Accepted for publication: 23 April 2014.

This work is licensed under a Creative Commons Attribution NonCommercial 3.0 License (CC BYNC 3.0).

(C) Copyright H. Lee et al., 2014

Licensee PAGEPress srl, Italy

International Journal of Plant Biology 2014; 5:4887 doi:10.4081/pb.2014.4887

relative size of its pulvinus, and ease in growing samples. After profiling the pulvinus proteome, it was compared to previously identified proteomes of nearby leaf tissue as well as guard cells, which are functionally similar to pulvini in that they also change cell shape through differences in turgor.

\section{Materials and Methods}

\section{Plant material}

Soybean ( $G$. $\max$ cv. Clark) seeds were soaked overnight in tap water before they were planted in 6-inch pots (2-3 per pot) with an $\mathrm{LCl}$ soil mixture (Sun Gro Horticulture, Vancouver, BC, Canada). The plants were grown in a growth chamber at the University of Maryland, College Park, set to a 16:8 photoperiod, temperatures of 25:20 C day:night, with a PAR of $500 \mathrm{~mol} \mathrm{~m}^{-2} \mathrm{~s}^{-1}$ and $60 \%$ relative humidity. The plants were watered to avoid water stress and received 100 ppm 20:20:20 fertilizer once a week. The plants were harvested after the appearance of six or seven trifoliolate leaves (between six and eight weeks). The terminal and lateral pulvini from the second through sixth trifoliolate leaves were separately excised with a razor one to two hours into the light period and frozen in liquid nitrogen. The pulvini were stored in $\mathrm{a}-80^{\circ} \mathrm{C}$ freezer until further use. 


\section{Protein extraction}

Trichloroacetic acid (TCA)/acetone precipitation, described previously by Natarajan et al. was used to extract pulvinar protein. ${ }^{17}$ For each of three biological replicates approximately $2.0 \mathrm{~g}$ of pulvinus were ground into a powder using a mortar and pestle with liquid nitrogen, then extracted with a $10 \%$ TCA $(\mathrm{w} / \mathrm{v}) / 0.07 \% \beta$-mercaptoethanol $(\mathrm{v} / \mathrm{v})$ in acetone mixture. Following a minimum of one hour incubation at $-20^{\circ} \mathrm{C}$ and centrifugation at $14,000 \mathrm{~g}$ in $4^{\circ} \mathrm{C}$ for 20 minutes, the supernatant was discarded. The pellet was rinsed with $0.07 \% \beta$-mercaptoethanol in acetone solution followed by centrifugation at $14,000 \mathrm{~g}$ $\left(4^{\circ} \mathrm{C}\right)$ for 20 minutes; the rinsing and centrifugation steps were repeated until the supernatant was clear. After vacuum drying, the pellet was resolubilized in a $7 \mathrm{M}$ urea, $2 \mathrm{M}$ thiourea, 4\% CHAPS, 1\% DTT solution and sonicated on ice for 45 minutes. The supernatant was collected after centrifugation at $14,000 \mathrm{~g}\left(4^{\circ} \mathrm{C}\right)$ for 20 minutes and the protein concentration was quantified using the Bradford method. ${ }^{18}$

\section{D gel electrophoresis}

For first dimension electrophoresis, $500 \mu \mathrm{g}$ of protein in a solution of $7 \mathrm{M}$ urea, $2 \mathrm{M}$ thiourea, 4\% CHAPS, $50 \mathrm{mM}$ DTT, 1\% IPG buffer (pH 4-7), and $0.002 \%$ bromophenol blue were loaded onto $13 \mathrm{~cm} \mathrm{IPG} \mathrm{strips,} \mathrm{pH} \mathrm{4-7,} \mathrm{and}$ run on a flatbed Ettan IPGphor II (GE Healthcare, Piscataway, NJ, USA) under conditions described earlier by Natarajan et al.: $30 \mathrm{~V}$ for 13 hours, $500 \mathrm{~V}$ for one hour, $1000 \mathrm{~V}$ for one hour, $8000 \mathrm{~V}$ gradually for 1:30 hours, $8000 \mathrm{~V}$ for $24000 \mathrm{Vhr}$, and $5000 \mathrm{~V}$ for ten hours. ${ }^{17} \mathrm{The}$ final step was truncated if the protein appeared to be sufficiently separated. Prior to second dimension SDS-PAGE the IPG strips were equilibrated twice to reduce the disulfide bridges, first in DTT and then in iodoacetamide (IAA), 15 minutes each in equilibration buffer (50 mM Tris-HCl pH 8.8, $6 \mathrm{M}$ urea, $30 \%$ glycerol, $2 \%$ SDS, $0.002 \%$ bromophenol blue, $1 \%$ DTT). The strips were loaded onto $12.5 \%$ polyacrylamide gels using a Hoefer SE 600 Ruby electrophoresis unit and run for 15 $\mathrm{mA}$ per gel for 30 minutes and $25 \mathrm{~mA}$ per gel for up to five hours. The gels were stained for two days using Coomassie Blue G-250. Gels were scanned on an ImageScanner III (GE Healthcare), and analyzed using Progenesis SameSpots software (Nonlinear Dynamics, Durham, NC, USA). Gels were stored in a $17.5 \%$ aqueous ammonium sulfate solution until further use.

\section{In-gel digestion}

Trypsin digestion of selected spots was based on methods by Shevchenko et al. (1996) and Gharahdaghi et al. (1999). ${ }^{19,20}$ Spots were excised and rinsed twice with 50\% methanol, ten minutes each. The gel pieces were reconstituted and subsequently dehydrated in solutions of $25 \mathrm{mM}$ ammonium bicarbonate and acetonitrile respectively, by placement on a shaker for ten minutes per solution. The prior step was repeated for a second time. The spots were further dried in a speed vac concentrator for about 15 minutes. Each gel piece was then reswollen with a $20 \mu \mathrm{L}$ aliquot of $10 \mathrm{ng} / \mu \mathrm{L}$ porcine trypsin (sequencing grade, Promega, Madison, WI, USA) in $25 \mathrm{mM}$ ammonium bicarbonate and refrigerated for one hour in $4^{\circ} \mathrm{C}$ before overnight incubation at $37^{\circ} \mathrm{C}$.

The excess trypsin solution surrounding the gel spot was transferred to new tubes and the remaining peptides were extracted from the gel pieces by $50 \%$ acetonitrile $/ 5 \%$ trifluoroacetic acid (TFA)(v/v). $50 \mu \mathrm{L}$ of the extraction mixture was added to each gel piece and placed in a shaker for an hour. The supernatants were added to the original trypsin digests and the extraction was repeated once more with another $50 \mu \mathrm{L}$ aliquot. The supernatants were then dried for up to two hours on a speed vac concentrator. The peptides were solubilized in a $20 \mu \mathrm{L}$ solution containing $5 \%$ acetonitrile $/ 0.1 \%$ formic acid.

\section{Mass spectrometry}

The peptides were run through an LTQ Orbitrap XL hybrid linear ion trap Orbitrap mass spectrometer (ThermoFisher Scientific, San Jose, CA, USA) with reverse-phase chromatography on a $100 \times 0.18 \mathrm{~mm}$ BioBasic- 18 column and $3 \mu \mathrm{L} / \mathrm{min}$ flow rate. The 30 -minute linear gradient was $5-40 \%$ acetonitrile in a $0.1 \%$ formic acid solution. The resolution survey scan was over the range $400-1600 \mathrm{~m} / \mathrm{z}$ ( $4=30000$ at $\mathrm{m} / \mathrm{z} 400$ ) and for each polypeptide gel spot, the MS/MS spectra of the five most abundant ions were recorded. The electrospray voltage was $3.5 \mathrm{kV}$ with normalized collision energy set to $30 \%$ and a minimum ion count of 5000. Mascot Distiller version 2.3.00 was employed for producing searchable peak lists.

\section{Data analysis}

MS/MS data were analyzed by the Scaffold toolkit version 3 (Proteome Software, Portland, OR). Scaffold searches MS/MS data against several database search engines and computes a peptide probability incorporating similar results among the search engines. Based on the peptide distribution, a protein probability is computed and the peptides are identified as parts of the computed protein. ${ }^{21}$ The MS/MS data was searched against the UniProt Knowledgebase. The results were limited to $G$. max, with minimum values of two significant peptide matches, $80 \%$ peptide identification probability, and 95\% protein identification probability. Uncharacterized proteins were identified by examining the homologous pro- tein clusters at $100 \%, 90 \%$, and $50 \%$ homology as curated by UniRef. Proteins without a name at the $50 \%$ homology level remained uncharacterized.

Biological process gene ontology (G0) terms as listed in the Gene Ontology Annotation (GOA) Database, a collaboration with the UniProt Knowledgebase, were retrieved for the proteins and input into the agriG0 version 1.2 G0 analysis program (China Agricultural University, Beijing, China) for single enrichment analysis. For this analysis, the abundance of the GO terms found in identified pulvinar proteins were compared to a background list of soybean genes. Single enrichment analysis indicates the dominant biological processes in the examined tissue against the entire plant species. It is one of several available methods to compare the significance of each G0 term. In addition, the analysis used as a default, a hypergeometric statistical method with the BenjaminiYekutieli correction method and a minimum five terms for significance. ${ }^{22}$ The reference G0 term list was the soybean locus genome provided by Phytozome (US Department of Energy, Joint Genome Institute, Walnut Creek, CA, USA). G0 terms significantly present in higher numbers in the pulvinus tissue were discussed.

\section{Results and Discussion}

\section{Protein extraction, identification, and functional analysis}

To investigate pulvinus protein functional analysis, 2-DE was used to separate the extracted proteins. A total of 183 spots were then excised for LC-MS/MS analysis (Figure 1). MS/MS data run through Scaffold resulted in the identification of 195 proteins; 18 spots of the 183 total either did not contain any peptides or had a protein identification probability $<95 \%$. Fifty-five proteins were named directly as a result of the Scaffold analysis workflow. An additional 116 proteins were named through the UniRef sequence clusters at the $100 \%, 90 \%$, or $50 \%$ homology level. The remaining 24 proteins were not named even at the $50 \%$ homology level. Of the 195 proteins, 129 had G0 terms for biological processes. Protein or cluster names, UniProtKB accession numbers, UniRef accession numbers, molecular weight, corresponding gel spots, and genetic information are listed in Supplementary Table 1.

AgriG0 single enrichment analysis of 129 proteins compared to a background soybean genome locus identified 74 significant G0 terms (data not shown). Most of the 74 terms fell under three broad parent terms: nucle- 
obase, nucleoside, and nucleotide metabolic processes (23 child G0 terms), nitrogen compound metabolic processes (13 child G0 terms), and transport (11 child G0 terms). All terminal significant child G0 terms are listed in Supplementary Table 2. The top two significant biological process $\mathrm{GO}$ terms were related to proton transport. The third significant term was a negative regulator of proton transport (oxidative phosphorylation, G0:0006119). Photosynthesis and respiration were also enriched compared to the reference background.

\section{Proton transport}

The two most significant (lowest e-values) G0 terms were related to the transport of protons through hydrolysis and synthesis of ATP (Supplementary Table 2). This would indicate a higher demand for proton transport in the pulvinus relative to the overall soybean plant and confirms our understanding of turgor change in pulvinar motor cells. The changes in the different turgor pressures between the adaxial and abaxial motor cells that result in leaf movement are triggered by $\mathrm{K}^{+}$and $\mathrm{Cl}^{-}$flux across the plasma membranes. Proton concentrations dictate the level of activity in ion channels and consequent osmotic movement. ${ }^{7}$ Vacuolar $\mathrm{H}^{+}$-ATPases are believed to also assist in $\mathrm{Ca}^{2+}$ accumulation through the generation of the electrochemical gradient across the tonoplast. ${ }^{23}$ In their examination of apoplast $\mathrm{pH}$ levels in the nyctinastic $S$. saman pulvini, Lee and Satter confirmed that swelling of motor cells corresponded to proton extrusion, and the same light treatment resulted in opposing proton fluxes between the adaxial and abaxial motor cells. ${ }^{24}$ Okazaki found similar $\mathrm{pH}$ changes in $P$. vulgaris pulvini and noted that an increase of extracellular $\mathrm{pH}$ were accompanied by inhibition of plasma membrane $\mathrm{H}^{+}$-ATPase activity which would lead to turgor decrease. ${ }^{15}$ Unlike Lee and Satter, Okazaki found that blue light increased extracellular $\mathrm{pH}$ in both adaxial and abaxial protoplasts and speculated that the relative differences in turgor between the two regions resulted from the differences in the amount of blue light received. It is important to remember that the responses measured by the two groups of researchers were not the same; Lee and Satter were looking at the leaf movements of a nyctinastic plant while Okazaki was examining the heliotropic leaf movement of $P$. vulgaris. The apparent differences in $\mathrm{pH}$ response to blue light may be a result of a photoreceptive signaling pathway unique to heliotropic plants.

\section{Malate metabolism}

Malate metabolism was the fifth most-common significant G0 term as calculated by agriG0 (Supplementary Table 2). In the pulvi-

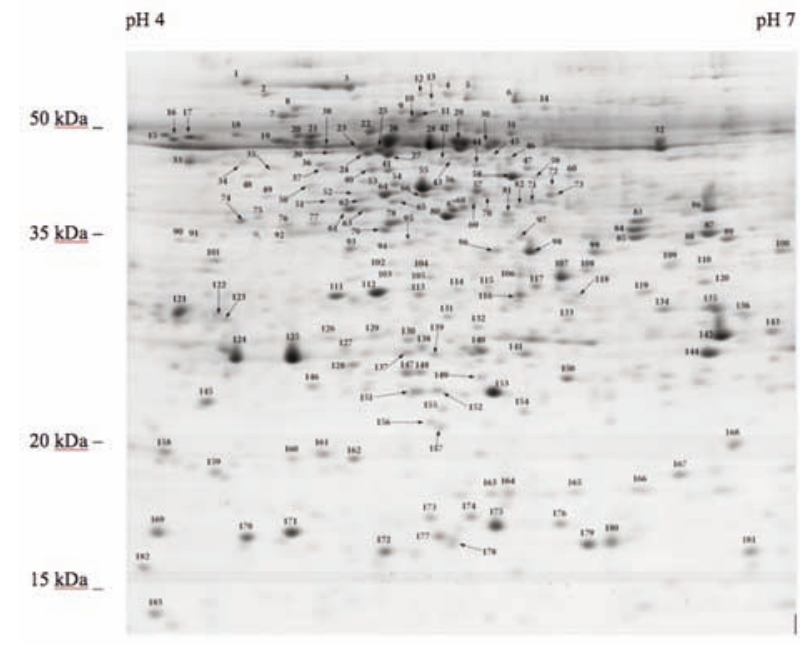

Figure 1. Representative SDS-PAGE gel of soybean lateral pulvinus, $\mathrm{pH}$ 4-7. The lateral and terminal pulvinus did not significantly differ from one another (data not shown). The numbers correspond to the proteins identified through LC-MS/MS and listed in Supplementary Table 1. Eighteen spots did not produce significant hits.

nus the dissociated anion of malic acid, malate, is believed to serve as an additional source of charge balance in the cell, as the cotransport of $\mathrm{Cl}^{-}$does not account for all the positive charge associated with $\mathrm{K}^{+}$movement. Malic acid appears in higher concentrations during the day in the whole pulvinus of $P$. coccineus motor cells than at night, and increased concentrations correspond to regions of $\mathrm{K}^{+}$ accumulation. The majority of malic acid occurs through de novo synthesis in situ ( $\sim 80 \%)$, not by transport between the abaxial and adaxial regions. ${ }^{25}$ Malate is also known to be an important counter-ion in guard cells. ${ }^{26}$ Four of the five proteins with the malate metabolism annotation were various isoforms of malate dehydrogenase (spots 83-85, 87). The fifth, malic enzyme (spot 13), is involved in the synthesis of malic acid. A precursor to malate is sucrose, believed to be present in motor cells by import from nearby tissues and also by the degradation of starch. One protein identified as the starch degrading enzyme beta-amylase was detected in various amounts across eight spots in the pulvinus gel (spots 15-17, 28-30, 51 and 62).

\section{Cytoskeletal processes}

Two significant $\mathbf{G O}$ terms, protein polymerization and microtubule-based movement, were both annotated to eight tubulin proteins (spots 18-21, 24, 25, 27, 38, 39 and 43). The multiple tubulin proteins are an example of the tetraploid nature of soybean; despite having over $86 \%$ sequence homology five tubulin beta2 chain proteins are encoded by genes located on five different chromosomes (data not shown). While tubulin is the cytoskeletal pro- tein type identified by significant G0 terms, studies have found actin (spots $53,55,57$ ) to be more integral to pulvinar bending. The G0 terms for actin proteins in the soybean pulvinus, however, have yet to be annotated and were therefore unable to be included in the analysis. Kanzawa et al. and Yao et al. detected the fragmentation of actin filaments in response to cold and electrical stimuli, respectively in Mimosa pudica pulvini; Kanzawa et al. also detected microtubule fragmentation but microtubule modulators did not appear to affect pulvinar bending while actin modulators did. ${ }^{1,27}$ Yao et al. noted that both actin rearrangement and the presence of intracellular $\mathrm{Ca}^{2+}$ were required for bending. ${ }^{27}$ Several other proteins with $\mathrm{Ca}^{2+}$-dependent activity were detected in the pulvinus, including inositol 3-phosphate synthase (spot 31), apyrase (spots 33 and 91), and annexin (spot 110). These three proteins could function as early participants in signal-transduction pathways that lead to ion movement across the plasma membrane.

\section{Ion transporters and aquaporins}

Along with proton ATPases was the expectation of finding aquaporins and $\mathrm{K}^{+}$transport proteins in the pulvinus. Previous studies have suggested the presence of aquaporins, and at least two $\mathrm{K}^{+}$transporters for inward and outward ion movement. ${ }^{28,29}$ The channels, conventionally labeled as $K_{H}$ and $K_{D}$ for hyperpolarized and depolarized $\mathrm{K}^{+}$channels, respectively, correspond to inward and outward ion movement. However, Yu et al. found evidence for a voltage-independent $\mathrm{K}_{\mathrm{H}}$ channel not affected by extracellular $\mathrm{pH}$ levels that appears to serve 
not a minor role in $\mathrm{K}^{+}$influx. ${ }^{30}$ Fluerat-Lessard et al. detected high levels of a $23 \mathrm{kDa}$ aquaporin in addition to $\mathrm{V}-\mathrm{H}^{+}$ATPases in the aqueous vacuole of mature $M$. pudica pulvini compared to juvenile, non-functioning pulvini. ${ }^{31}$ At least two aquaporin-like gene products were successfully cloned from $S$. saman pulvini; one of the two, SsAQP2, also demonstrated diurnal and circadian patterns and was not found to an appreciable degree in nearby leaf tissue. ${ }^{28}$ Unfortunately we did not detect any other transporters (other than ferritin, spot 147) or any proteins localized to the plasma membrane in the soybean pulvinus. There could be several explanations for the lack of these proteins. For one, aquaporins have an isoelectric point $(\sim 8)$ higher than the range that was used in this study. Furthermore, plasma membrane proteins typically are not recovered from traditional protein extraction methods, requiring an additional partitioning step and special extraction buffers. ${ }^{32}$ V-type $\mathrm{H}^{+}$-ATPases, such as the three detected in the soybean pulvinus, are not only localized in vacuoles but may also be located in the plasma membrane. The GO annotation for cellular component of the three $\mathrm{V}-\mathrm{H}^{+}$ATPases did not specify plasma membrane or the tonoplast; given the dearth of other plasma membrane proteins it seems more likely that the $\mathrm{H}^{+}$ATPases detected were from endomembranes.

\section{Pulvinus proteome compared to the root, leaf, and guard cell}

The pulvinus proteome profiled here was compared with the leaf proteome identified by $\mathrm{Xu}$ et al. to verify differences between the two tissues. ${ }^{33}$ One advantage of comparing the two tissues is that the protein extraction process as well as the soybean cultivar examined were the same in both studies. The distribution of pulvinar proteins in the various functional categories were expected to differ from the leaf because of the dominant biological functions distinct for type of tissue. Xu et al. identified a large number of soybean leaf proteins involved in energy production. ${ }^{33}$ Several of those proteins were also identified in the pulvinus, including rubisco large subunit, rubisco activase, and oxygen-evolving enhancer proteins 1 and 2, among others. The pulvinus proteome also contained ATP synthases and two other proteins involved in nucleoside phosphate biosynthesis which did not appear in the leaf proteome. While the separation methods for the two tissues were largely the same, Xu et al. used the method of Bevan et al. for classifying the proteins without reporting significant GO terms. ${ }^{34}$ As a result, direct side by side comparison of significant molecular processes cannot be undertaken. In general, the pulvinus proteome contained many more stress-response proteins than the leaf.
Pulvinus proteome compared to guard cells

Besides the pulvinus motor cell, another cell demonstrating rapid turgor changes is the guard cell. Like the pulvinus, guard cells require $\mathrm{H}^{+}$-ATPase activity triggered by light for its ion transport and subsequent water movement. In guard cells $\mathrm{H}^{+}$-ATPase activity in the plasma membrane increases when exposed to blue light, with a corresponding decrease of extracellular $\mathrm{pH}$ and turgor increase. ${ }^{26}$ However the opposite response occurred in Okazaki's examination of $P$. vulgaris motor cells, where blue light illumination led to the inhibition of $\mathrm{H}^{+}$-ATPase. ${ }^{15}$ This suggests that, despite functional similarities, guard cells and heliotropic pulvinus motor cells have distinct mechanisms for their turgor responses. It could be that guard cells, more often located on the abaxial leaf surface, have a similar photoreceptive mechanism to abaxial motor cells of nyctinastic plants such as $S$. saman. Therefore comparing the proteomes of the two tissues could assist in determining the degree of similarity of guard cells to pulvini of either heliotropic or nyctinastic plants.

Zhao et al. examined the diploid Arabidopsis guard cell proteome. ${ }^{35}$ Their top G0 term was related to stress response (response to cold), with energy categories making up three of the top eight. In the soybean pulvinus G0 terms for photosynthesis and respiration were less significant than in the Arabidopsis guard cell. The most abundant protein found in the Arabidopsis guard cell was the stress response protein TGG1, which functions as a defense against pathogen attack. ${ }^{35}$ In the soybean pulvinus protein abundance was not measured and so no similar observations could be made. The different rankings of significant G0 terms may reflect different mechanisms between the two. Zhu et al. compared mesophyll and guard cells from the tetraploid crop species Brassica napus using a combination of quantitative $\mathbf{G O}$ analysis and a qualitative functional approach following the functional categories listed by Bevan et al. for the Arabidopsis genome. ${ }^{34,36}$ Zhu et al. found the most highly enriched guard cell proteins relative to the mesophyll cell fell under the categories of energy, photosynthesis, membrane and transport, metabolism, and stress response. Categorization of the soybean pulvinus proteins using the Bevan et al. method found similar results for the major groups (data not shown). In contrast to the guard cell proteome, cytoskeletal and other structural proteins appeared to factor in more strongly in the pulvinus. Both Zhao et al. and Zhu et al. recovered around 10-fold more proteins than recovered in soybean pulvinus proteins, including many of the plasma membrane proteins that were not extracted using the methodology of this study. The magnitude of detected proteins in the guard cells compared to the pulvinus conceivably skews the G0 analysis. Additional comparisons of the two proteomes would require a higher amount of protein extraction from soybean pulvinus, and care must be taken to compare the soybean pulvinus with the guard cell profile of another tetraploid organism.

\section{Conclusions}

To our knowledge, this is a novel report on the analysis of protein in soybean pulvinus. These findings provide a better understanding of the molecular basis of pulvinar protein function. In summary, 195 proteins were extracted and positively identified from 165 spots from the pulvinus gel. Gene ontology analysis of significant terms found the top dominant GO biological processes were related to proton transport, malate metabolism, and cytoskeletal movement, which are in good agreement with previous studies on pulvinar response. Regrettably many highly basic proteins were not detected, including proteins at isoelectric points above $\mathrm{pH} 7$ and plasma membrane proteins. In order to recover the latter, methods such as two-phase partitioning and the use of special buffers would be necessary to recover the integral membrane proteins. Finally, the number of proteins recovered from the pulvinus will have to increase for future comparative studies with proteins of the guard cell.

\section{References}

1. Kanzawa N, Hoshino Y, Chiba M, et al. Change in the actin cytoskeleton during seismonastic movement of Mimosa pudica. Plant Cell Physiol 2006;47:531-9.

2. Herrera R, Krier C, Lalanne C, et al. Keeping the stem straight: a proteomic analysis of maritime pine seedlings undergoing phototropism and gravitropism. BMC Plant Biol 2010;10:217.

3. Zhang YL, Zhang HZ, Feng GY, et al. Leaf diaheliotropic movement can improve carbon gain and water use efficiency and not intensify photoinhibition in upland cotton (Gossypium hirsutum L.). Photosynthetica 2009;47:609-15.

4. Rakocevic M, Neumaier N, Oliveira GM, et al. Heliotropic responses of soybean cultivars at three phenological stages and under two water regimes. Pesqui Agropecu Bras 2010;45:661-70.

5. Kao WY, Forseth IN. Diurnal leaf movement, chlorophyll fluorescence and carbon assimilation in soybean grown under different nitrogen and water availabilities. Plant Cell Environ 1992;15:703-10. 
6. Grant RH, Potential effect of soybean heliotropism on ultraviolet-B irradiance and dose. Agron J 1999;91:1017-23.

7. Satter RL, Applewhite PB, Galston AW. Rhythmic potassium flux in Albizzia Effect of aminophylline, cations, and inhibitors of respiration and protein-synthesis. Plant Physiol 1974;54:280-5.

8. Ehleringer J, Forseth I. Solar tracking by plants. Science 1980;210:1094-8.

9. Dayanandan P, Hebard FV, Baldwin VD, Kaufman PB. Structure of gravity-sensitive sheath and internodal pulvini in grass shoots. Am J Bot 1977;64:1189-99.

10. Palmer JH, Asprey GF. Studies in the nyctinastic movement of the leaf pinnae of Samanea Saman (Jacq) Merrill .1. A general description of the effect of light on the nyctinastic rhythm. Planta 1958;51:757-69.

11. Rodrigues TM, Machado SR. Pulvinus functional traits in relation to leaf movements: a light and transmission electron microscopy study of the vascular system. Micron 2008;39:7-16.

12. Toriyama H. Distribution of tannin vacuoles in main pulvinus of mimosa pudica L. Proc Jpn Acad 1967;43:384-8.

13. Moysset L, Simon E. Secondary pulvinus of robinia-pseudoacacia (leguminosae) structural and ultrastructural features. Am J Bot 1991;78:1467-86.

14. Cronlund SL, Forseth IN. Heliotropic leaf movement response to $\mathrm{H}^{+} /$ATPase activation, $\mathrm{H}^{+} / \mathrm{ATPase}$ inhibition, and $\mathrm{K}^{+}$channel inhibition in vivo. Am $\mathrm{J}$ Bot 1995; $82: 1507-13$

15. Okazaki Y. Blue light inactivates plasma membrane $\mathrm{H}^{+}$)-ATPase in pulvinar motor cells of Phaseolus vulgaris L. Plant Cell Physiol 2002;43:860-8.

16. Clore AM, Turner WS, Morse AM, Whetten RW. Changes in mitogen-activated protein kinase activity occur in the maize pulvinus in response to gravistimulation and are important for the bending response. Plant Cell Environ 2003;26:991-1001.

17. Natarajan S, Xu C, Caperna TJ, Garrett WM. Comparison of protein solubilization methods suitable for proteomic analysis of soybean seed proteins. Anal Biochem 2005;342:214-20.

18. Bradford MM. Rapid and sensitive method for quantitation of microgram quantities of protein utilizing principle of protein-dye binding. Anal Biochem 1976;72:248-54.

19. Shevchenko A, Wilm M, Vorm O, Mann M. Mass spectrometric sequencing of proteins silver-stained polyacrylamide gels. Anal Chem 1996;68:850-8.

20. Gharahdaghi F, Weinberg CR, Meagher DA, et al. Mass spectrometric identification of proteins from silver-stained polyacrylamide gel: a method for the removal of silver ions to enhance sensitivity. Electrophoresis 1999;20:601-5.

21. Searle B. Scaffold: a bioinformatics tool for validating MS/MS-based proteomic studies. Proteomics 2010;10:1265-9.

22. Du Z, Zhou X, Ling Y, et al. agriG0: a G0 analysis toolkit for the agricultural community. Nuclei Acid Res 2010;38:W64-70.

23. Sze H, Li X, Palmgren MG. Energization of plant cell membranes by $\mathrm{H}^{+}$-pumping ATPases: regulation and biosynthesis. Plant Cell 1999;11:677-89.

24. Lee YS, Satter RL. Effects of white, blue, red-light and darkness on $\mathrm{Ph}$ of the apoplast in the Samanea pulvinus. Planta 1989;178:31-40.

25. Bialczyk J, Lechowski Z. Diurnal changes in the malic acid concentration in phseolus coccineus L. Pulvini. Plant Cell Physiol 1986;27:981-7.

26. Lawson T. Guard cell photosynthesis and stomatal function. New Phytol 2009; 181:13-34.

27. Yao $\mathrm{H}, \mathrm{Xu} \mathrm{Q}$, Yuan M. Actin dynamics mediates the changes of calcium level dur- ing the pulvinus movement of mimosa pudica. Plant Signal Behav 2008;3:954-60.

28. Moshelion M, Becker D, Czempinski K, et al. Diurnal and circadian regulation of putative potassium channels in a leaf moving organ. Plant Physiol 2002;128:634-42.

29. Moran N, Ehrenstein G, Iwasa K, et al. potassium channels in motor cells of samanea saman, a patch-clamp study. Plant Physiol 1988;88:643-8.

30. Yu L, Moshelion M, Moran N. Extracellular protons inhibit the activity of inward-rectifying potassium channels in the motor cells of Samanea saman pulvini. Plant Physiol 2001;127:1310-22.

31. Fleurat-Lessard P, Bouche-Pillon S, Leloup C, Bonnemain JL. Distribution and activity of the plasma membrane $\mathrm{H}^{+}$-ATPase in Mimosa pudica $\mathrm{L}$ in relation to ionic fluxes and leaf movements. Plant Physiol 1997;113:747-54.

32. Chevalier F. Highlights on the capacities of Gel-based proteomics. Proteome Sci 2010;8:23.

33. Xu C, Garrett WM, Sullivan J, et al. Separation and identification of soybean leaf proteins by two-dimensional gel electrophoresis and mass spectrometry. Phytochemistry 2006;67:2431-40.

34. Bevan M, Bancroft I, Bent E, et al. Analysis of $1.9 \mathrm{Mb}$ of contiguous sequence from chromosome 4 of arabidopsis thaliana. Nature 1998;391:485-8.

35. Zhao ZX, Zhang W, Stanley BA, Assman SM. Functional proteomics of arabidopsis thaliana guard cells uncovers new stomatal signaling pathways. Plant Cell 2008; 20:3210-26.

36. Zhu MM, Dai SJ, McClung S, et al. functional differentiation of brassica napus guard cells and mesophyll cells revealed by comparative proteomics. Mol Cell Proteomics 2009;8:752-66. 